\title{
Análisis multivariado de la supervivencia y los factores pronósticos de una cohorte de paciente con cáncer de mama receptor hormonal positivo
}

\author{
Analysis multivariate of survival and prognostic factors in a cohort of patients
} with hormone receptor-positive breast cancer

- Pedro Luis Ramos', María Athenas Ramos', Mario Arturo González'1,2, Mauricio García1,3

Centro de investigaciones clínicas Oncocare.

.

3 Instituto Nacional de Cancerología. Grupo Cirugía de Tejidos Blandos.

\section{Resumen}

Objetivo: el propósito de este estudio fue evaluar los factores pronósticos asociados con la supervivencia global y libre de enfermedad en pacientes con cáncer de mama receptor hormonal positivo.

Métodos: este estudio realizó el análisis de información de la clínica Oncocare en el período comprendido entre el $1^{\circ}$ de enero de 2003 hasta el 31 de julio de 2012. Se incluyeron todas las pacientes con diagnóstico de cáncer de mama receptor hormonal positivo (estado I a IIIC). Se construyeron curvas de supervivencia por el método de Kaplan-Meier, se compararon con el método de logaritmos y se efectuó un análisis de regresión de rangos proporcionales de Cox.

Resultados: un total de 387 pacientes fueron identificadas en el estudio. La tasa de mortalidad de la cohorte fue del 7,24\% y recaídas del 9,82\%. La edad media fue de 55,3 años y la mediana de seguimiento fue 48,2 meses.

El estado clínico se distribuyó en estado I con el 10,85\%, IIA con el 16,28\%, IIB con el 17,05\%, IIIA con el 22,74\%, IIIB con el $19,12 \%$ y IIIC con un 13,95\%. Los receptores de HER2 fueron positivos en un $13,7 \%$. Las pacientes recibieron quimioterapia neoadyuvante en un 60,72\%; el tipo de quimioterapia neoadyuvante fue AC en el 59,15\% y ACT en el 22,25\%. Se les administró quimioterapia adyuvante al 73,90\%: AC al 44,91\%, trastuzumab al 14,7\% y taxanos al 23,25\%. Las respuestas a la quimioterapia neoadyuvante fueron: respuesta objetiva en un $76,17 \%$, respuesta completa en un $14,89 \%$, respuesta parcial en un $61,28 \%$, y se presentaron recaídas en un $9,82 \%$ (locales $2,58 \%$, regionales $1,29 \%$ y sistémicas $7,75 \%$ ). En el análisis multivariado se evidencia que el número de ganglios positivos de 10 o más disminuye la supervivencia libre de enfermedad HR 2,30 (1,39-2,80). La respuesta clínica objetiva, el tamaño tumoral, HER2 positivo, receptor hormonal positivo, edad y estado clínico no modifican la supervivencia libre de enfermedad y global en el análisis multivariado.

Conclusión: los tiempos de supervivencia libre de enfermedad se disminuyen con 10 o más ganglios positivos; las otras variables analizadas: tamaño tumoral, estado clínico, edad, HER2 positivo y respuesta clínica objetivas, no afectan la supervivencia global y libre de enfermedad.

Palabras clave: cáncer de mama, pronóstico, receptor hormonal.

\begin{abstract}
Objective: The main purpose of this investigation was to evaluate prognosis factors associated with the overall and desease free survival of hormone receptor-positive breast cancer.

Methods: The data for this investigation was collected from the hospital Oncocare, from patients that were diagnosed with hormone receptor-positive breast cancer (stage I to IIIC) during the period of January 1st, 2003 to July 31st, 2012. This information was first used to construct survival curves using the Kaplan-Meier methods, then compared by using range logarithms, they were analysed using proportional range of regression by Cox.

Results: In this investigation, a total of 387 patients were used, the mortality rate for the cohort was $7.24 \%$, and relapses were $9.82 \%$. The median age was 55.3 years old, and the median follow-up were 48.2 months. The clinical stage distribution were as it follows, stage I 10.85\%, IIA 16.28\%, IIB $17.05 \%$, IIIA $22.74 \%$, IIIB $19.12 \%$ and IIIC $13.95 \%$; the HER2
\end{abstract}


receptors are positive by $13.7 \%$. The percentage of patients that received neoadjuvant chemotherapy was $60.72 \% ; 59.15 \%$ were type AC and $22.25 \%$ were type ACT. Additionally, $73.90 \%$ received adjuvant chemotherapy: type AC $44.91 \%$, trastuzumab $14.7 \%$ and taxenes $23.25 \%$. The response to neoadjuvant chemotherapy were: objective response $76.17 \%$, complete $14.89 \%$, partial $61.28 \%$; relapse were $9.82 \%$ (local 2.58\%, regionals $1.29 \%$ and systematic $7.75 \%$ ). Multivariate analysis also suggests that the number of positive lymph nodes, 10 or more, decrease desease free survival HR 2.30 (1.39-2.80). The clinical objective response, the tumour size, HER2 positive, positive hormonal receptors, age and clinical stage do not affect overall and desease free survival in the multivariate analysis.

Conclusion: The desease free survival decreases with 10 or more positive lymph nodes. Other variables such as tumour size, clinical stage, age, HER2 positive and objective clinical response do not affect overall and desease free survival.

Key words (MeSH): breast neoplasms, prognosis, hormonal receptors.

\section{Introducción}

Según la información de incidencias y mortalidad de Globocan del período 2012, el cáncer de mama es el segundo más frecuente en el mundo después del de pulmón y el más usual en mujeres, con un estimado de 1.670 .000 casos nuevos diagnosticados en 2012. La incidencia cruda fue de 47,7/100.000 y ajustada de 43,1/100.000. En cuanto a mortalidad, es la quinta causa de muerte por cáncer con 524.556 casos; la mortalidad cruda fue de 14,9/100.000 y ajustada de 12,9/100.000, y es la causa más frecuente de muerte por cáncer en mujeres en países subdesarrollados (324.000, 14,3\% del total). En Colombia, se presentaron 8.686 casos nuevos de cáncer de mama, que corresponden a una incidencia cruda del 35,9 y ajustada del 35,7/100.000, y se presentaron 2.649 muertes, para una mortalidad cruda de 11/100.000 y ajustada de $10,8 / 100.000^{1,2}$.

El estado de la enfermedad al diagnóstico es un factor determinante en el pronóstico de esta; desafortunadamente en países en desarrollo la mayoría de las pacientes se presentan con tumores localmente avanzados ${ }^{3,4}$ y Colombia no es la excepción. Un estudio realizado con 1.106 pacientes con cáncer de mama atendidas en diferentes instituciones de Bogotá mostró que los estados avanzados se presentan con mayor frecuencia, con un $63,8 \%{ }^{5}$, datos que son similares a los reportados por el Instituto Nacional de Cancerología (INC) en 2010, siendo los estados avanzados los más usuales ${ }^{6}$. Sin embargo, se observan diferencias de acuerdo con la población atendida: en 2005 una institución privada de Bogotá reportó que el estado al diagnóstico fue un $50,8 \%$ temprano ${ }^{7}$.

Son pocos los estudios efectuados en Colombia que muestren resultados a largo plazo en mujeres con cáncer de mama ${ }^{7,8}$; y la información para pacientes con receptor hormonal positivo es aún más escasa, si no inexistente. Este estudio tiene como objetivo mostrar la supervivencia libre de enfermedad y la supervivencia global, y los factores pronósticos asociados en una cohorte de mujeres con cáncer de mama receptor hormonal positivo atendidas en la clínica oncológica Oncocare durante el período 2003-2012.

\section{Materiales y métodos}

\section{Población}

Durante el período 2003-2012, se siguió una cohorte de 387 mujeres con cáncer de mama receptor hormonal positivo atendidas en la clínica oncológica Oncocare. Los análisis se realizaron aplicando el protocolo institucional que correspondió a seguimientos cada seis meses durante los cinco primeros años y luego anualmente. Durante los seguimientos a las mujeres, se llevaba a cabo examen clínico y mamografía anual, y de acuerdo con los hallazgos clínicos, se realizaban estudios complementarios para verificar recaídas de la enfermedad. Para documentar el estado vital de las mujeres que se perdieron del seguimiento, se hicieron llamadas telefónicas por la trabajadora social o a través de la página de web del Fosyga y de la Secretaría Distrital de Salud de Bogotá.

\section{Diseño}

Se trata de un estudio de cohorte bidireccional con un componente retrospectivo y prospectivo. Las pacientes que ingresaron desde el $1^{\circ}$ enero de 2003 hasta el 31 de diciembre de 2007 corresponden al componente retrospectivo; las que ingresaron a la cohorte a partir del $1^{\circ}$ de enero de 2008 hasta el 31 julio de 2012, al componente prospectivo.

\section{Objetivo principal}

Determinar la supervivencia global de las mujeres con cáncer de mama receptor hormonal positivo. 


\section{Objetivos secundarios}

1. Determinar la supervivencia libre de enfermedad de las mujeres con cáncer de mama receptor hormonal positivo.

2. Establecer la asociación entre los factores pronósticos, como estado ganglionar, receptores hormonales, receptor HER2, tamaño tumoral, estado clínico, respuesta objetiva y edad, con la supervivencia global de las mujeres con cáncer de mama receptor hormonal positivo.

3. Determinar la asociación entre los factores pronósticos, como estado ganglionar, receptores hormonales, receptor HER2, tamaño tumoral, estado clínico respuesta objetiva y edad, con la supervivencia libre de enfermedad de las mujeres con cáncer de mama receptor hormonal positivo.

\section{Resultados medidos}

Se calcularon la supervivencia libre de enfermedad y global. La supervivencia libre de enfermedad se definió como el tiempo desde el diagnóstico hasta la primera recaída o muerte por alguna causa. La supervivencia global se definió como el tiempo desde el diagnóstico hasta la muerte por cualquier causa.

Se determinó la respuesta clínica siguiendo los criterios

Recist. Estos se han adaptado del original del manual de la $\mathrm{OMS}^{17}$ teniendo en cuenta la medición del diámetro más largo para todas las lesiones blanco:

- Respuesta completa: la desaparición de todas las lesiones blanco.

- Respuesta parcial: disminución de al menos un $30 \%$ en la suma de los diámetros más largos de las lesiones blanco, tomando como referencia la suma de las línea de base.

- Enfermedad progresiva: por lo menos un aumento del $20 \%$ en la suma del diámetro más largo de las lesiones o la aparición de nuevas lesiones, una o más.

- Enfermedad estable: no hay disminución suficiente para calificar para respuesta parcial, ni aumento suficiente para calificar para una enfermedad progresiva $^{18}$. Las respuestas clínicas completas fueron confirmadas por patología.

\section{Análisis estadístico}

Se realizaron análisis descriptivos usando medidas de tendencia central, ubicación y dispersión para las variables continuas, y frecuencias absolutas y relativas para las variables categóricas. Se estimaron funciones de supervivencia (libre de enfermedad y global) utilizando el método no paramétrico de Kaplan-Meier, la cuales se compararon empleando la prueba de logaritmo del rango (log-rank). Se estimaron razones de peligro (HR) crudas y ajustadas usando regresión de Cox; para el ajuste se usaron las variables con resultados significativos en la prueba de log-rank. En los modelos de regresión de Cox, se verificó el supuesto de proporcionalidad; en los casos en que fue violado, la interacción entre la variable independiente y el tiempo fue introducida en el modelo. Los análisis se llevaron a cabo a dos colas para un nivel de error tipo I de 0,05. El programa estadístico Stata 11 fue utilizado para el análisis.

\section{Resultados}

Las características de las mujeres con cáncer de mama que participaron en la cohorte son descritas en la tabla 1. Un total de 387 pacientes fueron identificadas en el estudio. La edad media fue de 55,3 años. El estado clínico se distribuyó en estado I con el 10,85\%, IIA con el $16,28 \%$, IIB con el $17,05 \%$, IIIA con el $22,74 \%$, IIIB con el $19,12 \%$ y IIIC con el $13,95 \%$. El receptor de HER2 es positivo en un 13,7\%. Las pacientes recibieron quimioterapia neoadyuvante en un 60,72\%; el tipo de quimioterapia neoadyuvante fue $\mathrm{AC}$ en el $59,15 \%$ y $\mathrm{ACT}$ en el $22,25 \%$. Recibieron quimioterapia adyuvante en el $73,90 \%$, con AC en el $44,91 \%$, trastuzumab en el $14,7 \%$ y taxanos en el $23,25 \%$.

En la tabla 2 se muestra la respuesta clínica obtenida en las mujeres tratadas con quimioterapia neoadyuvante. Las respuestas a la quimioterapia neoadyuvante fueron: respuesta objetiva en un $76,17 \%$, respuesta completa en un $14,89 \%$ y respuesta parcial en un $61,28 \%$. La tasa de mortalidad de la cohorte fue del $7,24 \%$ y las recaídas de un 9,82\%.

Según el tipo de respuesta clínica, se encuentra que, en las mujeres que alcanzaron respuesta completa, el 98\% estaban libres de evento y las que no presentaron respuesta objetiva tuvieron un $40 \%$ de eventos de recaída o muerte (ver tabla 3).

Las funciones de supervivencia libre de enfermedad y global se ilustran en la figura 1 y 2 .

Los resultados del modelo de regresión de Cox para la supervivencia global se observan en la tabla 4. De las variables analizadas, el número de ganglios, las 


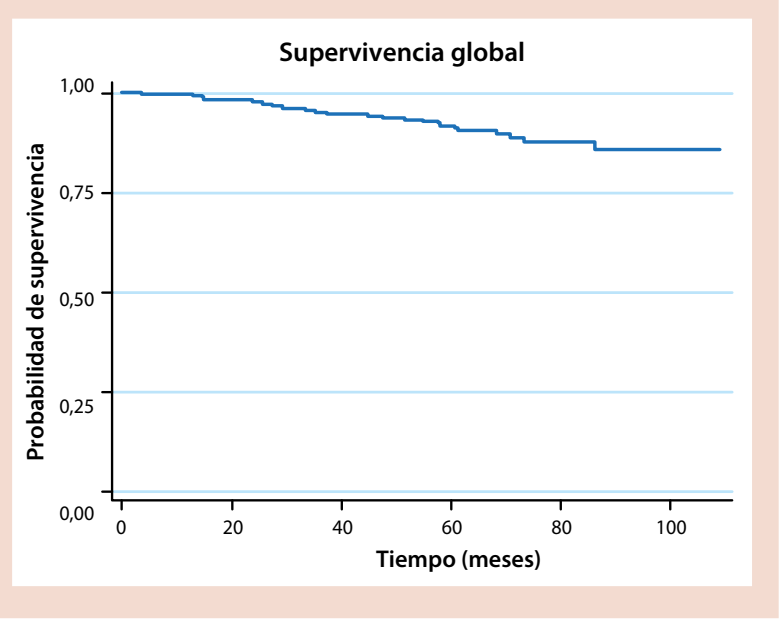

Figura 1. Supervivencia global de mujeres con cáncer de mama receptor hormonal positivo.

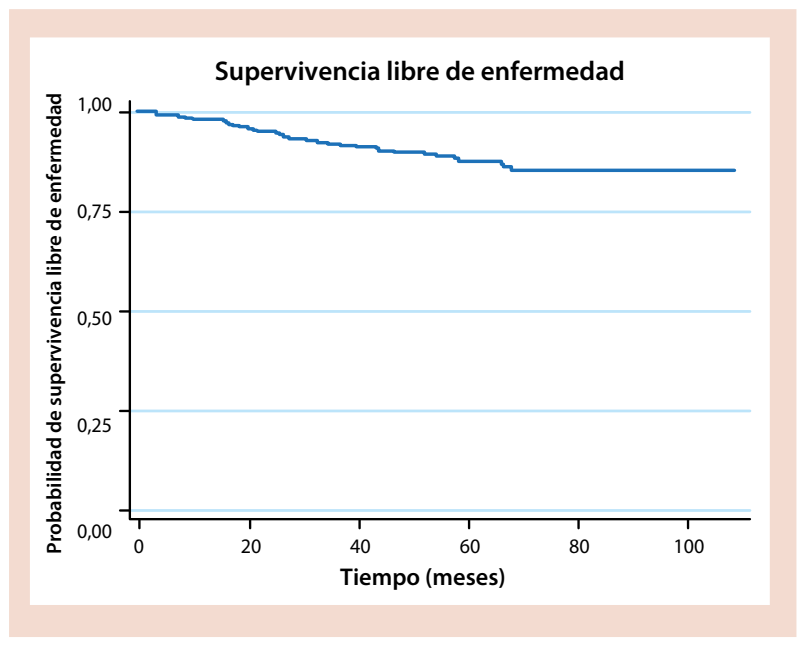

Figura 2. Supervivencia libre de enfermedad de mujeres con cáncer de mama receptor hormonal positivo.

respuestas clínicas objetivas (completas y parciales), HER2 positivo, edad, estado clínico y tamaño tumoral no modifican el riesgo de muerte.

En la tabla 5 se ven los resultados del modelo de Cox para la supervivencia libre de enfermedad. El número de ganglios positivos de 10 o más disminuye la supervivencia libre de enfermedad HR ajustado 2,30 $(1,39-2,80)$. Pero los otros factores analizados, respuesta clínica objetiva, tamaño tumoral, HER2 positivo, edad y estado clínico, no modifican la supervivencia libre de enfermedad.

\section{Discusión}

El objetivo del estudio fue analizar la supervivencia libre de enfermedad y global a largo plazo y los principales factores pronósticos asociados a estas, en una cohorte de mujeres con cáncer de mama receptor hormonal positivo diagnosticadas durante el período 2003-2012 que fueron tratadas en la clínica Oncocare de Bogotá (Colombia). A la mediana de seguimiento de 48 meses, el 90,18\% de las mujeres estaban libres de enfermedad y el $92,76 \%$ no habían muerto. El resultado para supervivencia fue similar al reportado para mujeres de Noruega diagnosticadas durante el período 19992000, el cual correspondió al 84\%9 y en los Estados Unidos al $89 \%{ }^{10}$.

En el contexto colombiano, un estudio reporta que la supervivencia global a cinco años en mujeres con cáncer temprano tratadas con cirugía conservadora y radioterapia fue del $92,9 \%{ }^{8}$. Otro estudio que analiza la supervivencia en una cohorte de 1.328 mujeres con cáncer de mama reporta que en los estados tempranos esta fue del $88,2 \%$ al $94,4 \% 7$, pero no se informa la supervivencia para el subgrupo específico de cáncer de mama receptor hormonal positivo.

De los factores pronósticos analizados, se encontró que el compromiso ganglionar masivo disminuye la supervivencia libre de enfermedad HR 2,30 (1,39-2,80) para 10 o más ganglios positivos, pero no fue significativo para supervivencia global en 10 o más ganglios $1,63(0,99-2,67)$. Otros factores descritos como factores pronósticos adversos fueron el tamaño tumoral mayor a $5 \mathrm{~cm}$, tener HER2 positivo y ser un cáncer avanzado al diagnóstico. Sin embargo, no fueron significativos para la supervivencia libre de enfermedad y global.

Estos resultados son similares a los reportados en la literatura, donde se encuentra que el número de ganglios positivos se asocian a tiempo de supervivencia libre de enfermedad menor ${ }^{7,8,11-13}$; el estado avanzado al diagnóstico, el tamaño tumoral y la expresión del receptor HER2+ reportados en la mayoría de los estudios relacionados con menor supervivencia libre de enfermedad y global no se asociaron en este estudio con estos desenlaces.

El estado de receptor HER2 positivo no se relacionó con riesgo de muerte y recaída con HR 1,09 (0,72-1,65) y $1,07(0,73-1,68)$ para supervivencia libre de enfermedad y global, respectivamente, lo cual es similar a los estudios publicados en este sentido, probablemente por el efecto benéfico de la quimioterapia con trastuzumab ${ }^{19}$.

Un estudio que muestra resultados a largo plazo en mujeres estado II/III que fueron tratadas con 
Tabla 1. Características de base

\begin{tabular}{|c|c|}
\hline Edad promedio (SD) & $55,3(11,53)$ \\
\hline Mujeres posmenopausia n (\%) & $253(65,37)$ \\
\hline \multicolumn{2}{|l|}{ Estadio clínico n (\%) } \\
\hline Temprano & $171(44,19)$ \\
\hline 1 & $42(10,85)$ \\
\hline$\| \mathrm{A}$ & $63(16,28)$ \\
\hline$\| \mathrm{B}$ & $66(17,05)$ \\
\hline Avanzado & $216(55,81)$ \\
\hline$\| A$ & $88(22,74)$ \\
\hline IIIB & $74(19.12)$ \\
\hline IIIC & $54(13,95)$ \\
\hline \multicolumn{2}{|l|}{ HER2 n (\%) } \\
\hline Positivo & $53(13,70)$ \\
\hline Negativo & $286(73,90)$ \\
\hline Indeterminado & $8(2,07)$ \\
\hline Sin información & $40(10,33)$ \\
\hline \multicolumn{2}{|l|}{ Receptor hormonal n (\%) } \\
\hline ER+/PgRt & $317(81,91)$ \\
\hline$E R+/ P g R-$ & $60(15,50)$ \\
\hline Sin información & $10(2,58)$ \\
\hline \multicolumn{2}{|l|}{ Tipo histológico n (\%) } \\
\hline Ductal & $342(88,37)$ \\
\hline Lobulillar & $26(6,72)$ \\
\hline Mixto & $8(2,07)$ \\
\hline Sin información & $11(2,84)$ \\
\hline \multicolumn{2}{|l|}{ Grado histológico n (\%) } \\
\hline Grado I & $38(9,82)$ \\
\hline Grado ॥ & $217(56,07)$ \\
\hline Grado III & $97(25,06)$ \\
\hline Sin información & $35(9,04)$ \\
\hline \multicolumn{2}{|l|}{ Tamaño tumoral n (\%) } \\
\hline Menor a $21 \mathrm{~mm}$ & $86(16,23)$ \\
\hline
\end{tabular}

Tabla 2. Respuesta clínica a la quimioterapia de acuerdo con los criterios Recist

\begin{tabular}{|l|c|c|c|c|}
\hline $\begin{array}{c}\text { Tipo de } \\
\text { quimioterapia } \\
\text { neoadyuvante } \\
\mathbf{n}(\%)\end{array}$ & Completa & Parcial & Estable & Progresión \\
\hline AC & $15(10,79)$ & $87(62,59)$ & $35(25,18)$ & $2(1,44)$ \\
\hline CMF & $2(7,14)$ & $19(67,89)$ & $6(21,43)$ & $1(3,57)$ \\
\hline ACT & $17(26,99)$ & $35(55,56)$ & $9(14,28)$ & $2(3,17)$ \\
\hline ACTH & $1(20,00)$ & $3(60,00)$ & $0(0,00)$ & $1(20,00)$ \\
\hline Total & $35(14,89)$ & $144(62,29)$ & $50(21,28)$ & $6(2,5)$ \\
\hline
\end{tabular}

quimioterapia reporta que para 10 años de seguimiento el $89,1 \%$ de las mujeres con buena respuesta (completa o parcial) estaban vivas comparado con el $55,5 \%$ de las mujeres que no respondieron (estable o progresión) ${ }^{14}$, pero en este análisis no hay asociación de la respuesta de la quimioterapia con la supervivencia libre de enfermedad y global.

\begin{tabular}{|c|c|}
\hline 21 a $50 \mathrm{~mm}$ & $222(41,89)$ \\
\hline Mayor a $50 \mathrm{~mm}$ & $222(41,89)$ \\
\hline \multicolumn{2}{|l|}{ Número de ganglios positivos $n(\%)$} \\
\hline Ninguno & $172(44,45)$ \\
\hline 1 a 3 & $81(20,93)$ \\
\hline $4 \mathrm{a} 9$ & $48(12,40)$ \\
\hline Más de 10 ganglios & $34(8,79)$ \\
\hline Sin información & $52(13,43)$ \\
\hline \multicolumn{2}{|l|}{ Estado funcional $\mathrm{n}(\%)$} \\
\hline ECOG 0 & $333(86,05)$ \\
\hline ECOG 1 & $53(13,70)$ \\
\hline ECOG 2 & $1(0,25)$ \\
\hline \multicolumn{2}{|l|}{ Tipo quimioterapia neoadyuvante n (\%) } \\
\hline AC & $139(59,15)$ \\
\hline CMF & $281(11,91)$ \\
\hline ACT & $63(26,82)$ \\
\hline ACTH & $5(2,13)$ \\
\hline \multicolumn{2}{|l|}{ Tipo quimioterapia adyuvante n (\%) } \\
\hline$A C$ & $128(44,91)$ \\
\hline CMF & $28(8,00)$ \\
\hline Taxanos & $109(31,05)$ \\
\hline Trastuzumab & $63(17,81)$ \\
\hline \multicolumn{2}{|l|}{ Seguridad social n (\%) } \\
\hline Contributivo & $297(56,04)$ \\
\hline Subsidiado & $233(43,96)$ \\
\hline \multicolumn{2}{|l|}{ Tratamiento recibido n (\%) } \\
\hline Tratamiento con quimioterapia adyuvante & $351(66,23)$ \\
\hline Tratamiento con quimioterapia neoadyuvante & $350(66,04)$ \\
\hline Tratamiento con hormonoterapia adyuvante & $357(67,36)$ \\
\hline Tratamiento con cirugía & $382(98,71)$ \\
\hline Cirugía conservadora & $192(50,26)$ \\
\hline Tratamiento con radioterapia adyuvante & $249(64,34)$ \\
\hline
\end{tabular}

Tabla 3. Eventos de recaída o muerte según la respuesta clínica

\begin{tabular}{|c|c|c|c|c|}
\hline & Completa & Parcial & Estable & Progresión \\
\hline Recaída n (\%) & & & & \\
\hline Local & $0(0,00)$ & $2(28,57)$ & $2(28,57)$ & $3(42,86)$ \\
\hline Regional & $0(0,00)$ & $2(40,00)$ & $2(40,00)$ & $1(20,00)$ \\
\hline Sistémica & $1(4,34)$ & $11(47,83)$ & $8(34,79)$ & $13(13,04)$ \\
\hline Total & $1(2,22)$ & $15(33,34)$ & $12(26,66)$ & $17(37,78)$ \\
\hline Muerte & $0(0,00)$ & $9(50,00)$ & $8(45,00)$ & $1(5,00)$ \\
\hline $\begin{array}{l}\text { Evento recaída } \\
\text { o muerte n (\%) }\end{array}$ & & & & \\
\hline Sin evento & $34(98,00)$ & $129(90,00)$ & $38(76,00)$ & $5(84,00)$ \\
\hline $\begin{array}{c}\text { Recaída } \\
\text { muerte }\end{array}$ & $1(2,00)$ & $15(10,00)$ & $12(24,00)$ & $1(16,00)$ \\
\hline
\end{tabular}

Este estudio tiene la limitación del poco número de mujeres con períodos de seguimiento largos (más de 100 meses), con lo cual el número de eventos no fue suficiente para realizar análisis más detallados de los factores pronósticos asociados a la supervivencia, 
Tabla 4. Resultados modelo de Cox para la supervivencia global

\begin{tabular}{|c|c|c|}
\hline Variable & $\begin{array}{l}\text { HR crudo } \\
\text { (IC 95\%) }\end{array}$ & $\begin{array}{l}\text { HR ajustado } \\
\text { (IC } 95 \%)\end{array}$ \\
\hline \multicolumn{3}{|l|}{ Estadio clínico } \\
\hline Temprano & 1 & 1 \\
\hline Avanzado & $1,17(0,95-1,43)$ & $0,86(0,57-1,31)$ \\
\hline \multicolumn{3}{|l|}{ HER2 } \\
\hline Negativo & 1 & 1 \\
\hline Positivo & $1,11(0,82-1,49)$ & $1,07(0,73-1,68)$ \\
\hline \multicolumn{3}{|l|}{ Tamaño tumoral } \\
\hline Menor a 21 mm & 1 & 1 \\
\hline 21 a $50 \mathrm{~mm}$ & $1,18(0,90-1,55)$ & $1,15(0,15-8,63)$ \\
\hline Mayor a 50 mm & $1,20(0,91-1,59)$ & $1,04(0,13-7,93)$ \\
\hline \multicolumn{3}{|l|}{ Edad } \\
\hline 50 años o menos & 1 & 1 \\
\hline Mayor a 50 años & $1,06(0,86-1,31)$ & $1,02(0,76-1,36)$ \\
\hline \multicolumn{3}{|l|}{ Ganglios positivos } \\
\hline Ninguno & 1 & 1 \\
\hline 1 a 3 & $0,99(0,76-1,30)$ & $0,89(0,59-1,36)$ \\
\hline 4 a 9 & $0,94(0,68-1,29)$ & $1,04(0,64-1,66)$ \\
\hline Más de 10 ganglios & $1,36(0,94-1,98)$ & $1,63(0,99-2,67)$ \\
\hline \multicolumn{3}{|l|}{ Respuestaf } \\
\hline No respuesta & 1 & 1 \\
\hline Respuesta & $1,35(1,00-1,84)$ & $1,34(0,99-2,30)$ \\
\hline
\end{tabular}

£ Para esta variable, respuesta corresponde a respuesta clínica completa o parcial y no a respuesta a enfermedad estable o progresión.

Tabla 5. Resultados modelo de Cox para la supervivencia libre de enfermedad

\begin{tabular}{|c|c|c|}
\hline Variable & $\begin{array}{l}\text { HR crudo } \\
\text { (IC } 95 \% \text { ) }\end{array}$ & $\begin{array}{l}\text { HR ajustado } \\
\text { (IC } 95 \%)\end{array}$ \\
\hline \multicolumn{3}{|l|}{ Estadio clínico } \\
\hline Temprano & 1 & 1 \\
\hline Avanzado & $1,17(0,96-1,43)$ & $0,72(0,47-1,10)$ \\
\hline \multicolumn{3}{|l|}{ HER2 } \\
\hline Negativo & 1 & 1 \\
\hline Positivo & $1,10(0,82-1,48)$ & $1,09(0,72-1,65)$ \\
\hline \multicolumn{3}{|l|}{ Tamaño tumoral } \\
\hline Menor a $21 \mathrm{~mm}$ & 1 & 1 \\
\hline 21 a $50 \mathrm{~mm}$ & $1,20(0,91-1,58)$ & $1,25(0,16-9,34)$ \\
\hline Mayor a $50 \mathrm{~mm}$ & $1,21(0,91-1,60)$ & $1,10(0,14-8,34)$ \\
\hline \multicolumn{3}{|l|}{ Edad } \\
\hline 50 años o menos & 1 & 1 \\
\hline Mayor a 50 años & $1,09(0,88-1,34)$ & $1,10(0,82-1,47)$ \\
\hline \multicolumn{3}{|l|}{ Ganglios positivos } \\
\hline Ninguno & 1 & 1 \\
\hline 1 a 3 & $1,06(0,82-1,39)$ & $1,04(0,68-1,57)$ \\
\hline 4 a 9 & $0,95(0,69-1,32)$ & $1,11(0,69-1,79)$ \\
\hline Más de 10 ganglios & $1,76(1,21-2,56)$ & $2,30(1,39-2,80)$ \\
\hline \multicolumn{3}{|l|}{ Respuesta⿷* } \\
\hline No respuesta & 1 & 1 \\
\hline Respuesta & $1,31(0,97-1,91)$ & $1,44(0,98-2,10)$ \\
\hline
\end{tabular}

$£$ Para esta variable, respuesta corresponde a respuesta clínica completa o parcial y no a respuesta a enfermedad estable o progresión.

* Esta variable no cumplió el supuesto de proporcionalidad. Se incluyó la interacción de esta variable con el tiempo en el modelo, la cual fue significativa (1,06 IC 95\% 1,018-1,12). Esto indica que el $\mathrm{HR}$ no es constante en el tiempo $\left(H R=0,05^{*}(1,06) \wedge t\right)$. En el modelo ajustado, la interacción también fue significativa $(1,06 \mid C$ $95 \%$ 1,001-1,12). por ejemplo, determinar el riesgo de acuerdo con la respuesta completa comparada con la parcial o con el estado del receptor HER2.

Se debe continuar con el seguimiento de esta cohorte de pacientes para determinar a largo plazo las supervivencias libres de enfermedad y global y los factores asociados a estas, de forma tal que se pueda contar con información que permita caracterizar mejor el riesgo de las mujeres colombianas con cáncer de mama y así poder ofrecer alternativas que mejoren el pronóstico de la enfermedad.

\section{Conclusión}

Los tiempos de supervivencia libre de enfermedad se disminuyen con el número de ganglios positivos de 10 o más; las otras variables analizadas: tamaño tumoral, estado clínico, edad, HER2, receptores hormonales y respuestas clínicas objetivas, no se asocian con la supervivencia global y libre de enfermedad.

\section{Referencias}

1. Instituto Nacional de Cancerología (INC), Instituto Geográfico Agustín Codazzi (IGAC). Atlas de mortalidad por cáncer en Colombia. 3a ed. Bogotá: Imprenta Nacional de Colombia; 2010.

2. International Agency for Research on Cancer. Globocan 2008. IARC 2012 [citado 2012 sep 15]. Disponible en: http://globocan.iarc.frl

3. Piñeros $M$, Sánchez $R$, Cendales $R$, et al. Características sociodemográficas, clínicas y de la atención de mujeres con cáncer de mama en Bogotá. Rev Colomb Cancerol 2008;12:181-90.

4. Schwartsmann G. Breast cancer in South America: challenges to improve early detection and medical management of a public health problem. J Clin Oncol 2001;19(18 Suppl):118S-24S.

5. Piñeros $M$, Sánchez R, Cendales R, Perry F, Ocampo R. Patient delay among Colombian women with breast cancer. Salud Pública Mex 2009;51(5):372-80.

6. Instituto Nacional de Cancerología (INC). Anuario estadístico 2010. Bogotá: Impreso en Buenos y Creativos SAS; 2012.

7. Robledo JF, Caicedo JJ, De Antonio R. Análisis de supervivencia en una cohorte de 1.328 pacientes con carcinoma de seno. Rev Colomb Cir 2005;20(1):4-20.

8. Ospino R, Cendales R, Sánchez Z, Bobadilla I, Galvis J, Cifuentes J. Supervivencia en pacientes con cáncer de mama temprano tratadas con cirugía conservadora asociada a radioterapia en el Instituto Nacional de Cancerología. Rev Colomb Cancerol 2011;15(2):21-30.

9. Kalager M, Haldorsen $T$, Bretthauer M, Hoff G, Thoresen SO, Adami HO. Improved breast cancer survival following introduction of an organized mammography screening program among both screened and unscreened women: a population-based cohort study. Breast Cancer Res 2009;11(4):R44. 
10. Sant M, Allemani C, Berrino F, Coleman MP, Aareleid T, Chaplain $\mathrm{G}$, et al. Breast carcinoma survival in Europe and the United States. Cancer 2004;100(4):715-22.

11. Giltnane JM, Moeder CB, Camp RL, Rimm DL. Quantitative multiplexed analysis of ErbB family coexpression for primary breast cancer prognosis in a large retrospective cohort. Cancer 2009;115(11):2400-9.

12. Guerra MR, Mendonca GA, Bustamante-Teixeira MT, Cintra JR, Carvalho LM, Magalhaes LM. [Five-year survival and prognostic factors in a cohort of breast cancer patients treated in Juiz de Fora, Minas Gerais State, Brazil]. Cad Saude Publica 2009;25(11):2455-66.

13. Mauriac L, Keshaviah A, Debled M, Mouridsen H, Forbes JF, Thurlimann B, et al. Predictors of early relapse in postmenopausal women with hormone receptor-positive breast cancer in the BIG 1-98 trial. Ann Oncol 2007;18(5):859-67.

14. Kim J, Lee J, Chang E, Suh $K$, Lee $C$, Jee J, et al. Prognostic factors in patients with stage ii/iii breast cancer treated with adjuvant extension of neoadjuvant chemotherapy: a retrospective cohort study with ten-years of follow-up data. J Breast Cancer 2011;14(1):39-45.
15. Dunnwald LK, Rossing MA, Li Cl. Hormone receptor status, tumor characteristics, and prognosis: a prospective cohort of breast cancer patients. Breast Cancer Res 2007;9(1):R6

16. Grann VR, Troxel AB, Zojwalla NJ, Jacobson JS, Hershman D, Neugut Al. Hormone receptor status and survival in a population-based cohort of patients with breast carcinoma. Cancer 2005; 103(11):2241-51.

17. WHO handbook for reporting results of cancer treatment. Geneva (Switzerland): World Health Organization Offset Publication $N^{\circ} 48 ; 1979$.

18. Therasse P, Arbuck SG, Eisenhauer EA, Wanders J, Kaplan RS, Rubinstein $\mathrm{L}$, et al. New guidelines to evaluate the response to treatment in solid tumors. European Organization for Research and Treatment of Cancer, National Cancer Institute of the United States, National Cancer Institute of Canada. J Natl Cancer Inst 2000;92(3):205-16.

19. Harris L, Fritsche $H$, Mennel $R$, Norton L, Ravdin P, Taube $S$, et al. American Society of Clinical Oncology 2007 update of recommendations for the use of tumor markers in breast cancer. J Clin Oncol 2007;25(33):5287-312. 